ALDO GORINI

Istituto Superiore di Scienze Religiose di Genova (Italia)

\title{
IL DIRITTO CANONICO NEL CONTESTO TEOLOGICO ED ECCLESIALE DEI SECOLI V-VIII
}

Sommario: Premessa. - 1. La teologia nei secoli V-VIII. - 2. Il diritto canonico nei secoli V-VIII: la Collectio Dionysiana, Gregorio Magno, i concili. - 2.1. Lopera di Dionigi il Piccolo. - 2.2. Gregorio Magno. - 2.3. I concili. - 3. Presenza del diritto e tendenze al giuridismo nella Chiesa dei secoli V-VIII: le regole monastiche, i penitenziali. - Osservazioni conclusive.

\section{Premessa}

Questo scritto prende avvio dalla constatazione che nei manuali di storia della teologia e in quelli di patrologia il diritto canonico è trascurato o toccato solo incidentalmente o preso in considerazione piuttosto settorialmente, mentre, per altro verso, i manuali di storia del diritto canonico ripetutamente si occupano sbrigativamente del periodo che va dal V all'VIII secolo e senza particolare attenzione per il contesto teologico generale. Gli approfondimenti monografici, poi, tendono a fissare l'obiettivo su punti circoscritti del panorama dell'epoca.

Vorrei, invece, accennare un confronto tra teologia, diritto canonico e vissuto ecclesiale dei secoli V-VIII, credendo che da uno studio del genere possano derivare utili risultati. Considerando il diritto canonico in comparazione con il contesto teologico ed ecclesiale mi sembra possano risaltare linee e caratteri significativi del periodo e inserendo il diritto canonico nel contesto teologico dell'epoca mi pare possano emergere più precisi equilibri descrittivi. Il mio intervento comunque offre solo alcune osservazioni e qualche possibile spunto di riflessione scientifica partendo semplicemente da dati storiografici. Ciò che presento, più che l'esito di una ricerca, è una proposta di 
ricerca, con un primo suggerimento di idee ed ipotesi attraverso la raccolta e collazione di alcuni elementi.

Riferendomi ai secoli V-VIII, richiamerò dapprima alcuni dati generali concernenti la storia della teologia, segnalerò quindi qualche elemento particolarmente espressivo per la storia del diritto canonico, metterò ancora in evidenza la presenza del diritto e di tendenze al giuridismo e arriverò infine ad osservazioni conclusive.

\section{La teologia nei secoli V-VIII}

Risulta anche dai più noti manuali di storia della Chiesa odierni che dopo il Concilio di Calcedonia (451) venne meno la grande fioritura della letteratura e della scienza ecclesiastica cominciata al principio del IV secolo con il riconoscimento del cristianesimo da parte dello Stato romano e si verificò complessivamente un affievolimento della forza creativa sia per la teologia greca sia per il campo teologico occidentale ${ }^{1}$. L'epoca che inizia alla metà del V secolo non può essere annoverata fra i grandi periodi della teologia classica ${ }^{2}$. Nell'esegesi biblica, nella teologia speculativa, nella storiografia si sviluppò la tendenza alla compilazione, che si manifestò con la forma caratteristica delle catenae e con la raccolta di flores, sententiae, excerpta ${ }^{3}$.

È già stato tuttavia affermato che la mancanza di una evidente creatività nella teologia del periodo non è da considerare semplicemente come decadenza ed è stato spiegato che in un certo senso la riflessione teologica è diventata più raffinata, più sottile, e che i teologi della cosiddetta età patristica posteriore, specialmente quelli del secolo VI, hanno posto le fondamenta per gli sviluppi successivi della teologia

\footnotetext{
${ }^{1}$ Cf., p. es., K. Bihlmeyer - H. Tuechle, Storia della Chiesa. I: L'antichità cristiana, ed. I. Rogger, Brescia $1986^{10}, 453-454,492,498$.

${ }^{2}$ Cf. H.-G. BECK, La Chiesa protobizantina, in: Storia della Chiesa, ed. H. Jedin. III: La Chiesa tra Oriente e Occidente. La Chiesa imperiale in Oriente, l'incontro della Chiesa con i barbari, il monachesimo latino (V-VII sec.), Milano 1978, 81; J. Vogt, Epilogo della letteratura paleocristiana latina, ibid., 390.

${ }^{3}$ Cf. L. Allevi, Disegno di storia della teologia, Torino 1939, 29-32.
} 
sia orientale sia occidentale". Si potrebbe forse dire che, "fisiologicamente", si è trattato anche di una fase di assestamento e di consolidamento seguita al precedente slancio, sebbene sia necessario tenere presenti pure vari fattori storici, come la trasmigrazione dei popoli in Occidente. La letteratura cristiana di transizione dalla tarda antichità al primo medioevo pare concepire se stessa come prosecuzione e parte dell'antica letteratura patristica, benché metta in evidenza, sia in Occidente che in Oriente, segni che denotano la fine di un'epoca e il passaggio ad una nuova era: nella tensione tra un grande passato e la consapevolezza di una veniente età nuova, tale letteratura si preoccupa innanzitutto di mantenere in vita le precedenti conquiste culturali e di trasmetterle alla posterità, inoltre assume il ruolo di offrire un orientamento alla nuova epoca che sorge, sia sul piano pratico che su quello filosofico e teologico ${ }^{5}$. I più eminenti autori del periodo furono anelli di congiunzione tra i Padri e gli scolastici ${ }^{6}$. Nella prima metà del VI secolo Leonzio di Bisanzio e Giovanni Filopono in Oriente e Boezio in Occidente misero la filosofia aristotelica, in particolare la logica, a servizio del pensiero teologico, ponendo così la base formale per il fiorire della scolastica medievale 7 . I teologi medievali, del resto, poterono anche adoperare le precedenti compilazioni ${ }^{8}$.

Comunque, per quanto concerne la teologia, l'epoca che va dal V all'VIII secolo ebbe evidentemente una minore dinamicità rispetto a quella immediatamente precedente, prevalsero indirizzi conservativi e ripropositivi, fu un periodo importante e anche di supporto, ma, nell'insieme, non nel senso dell'originalità. È stato scritto persino che «All'epoca d'oro dei secoli IV e V, segue un'epoca d'argento (sec. VI)

${ }^{4}$ Cf. B. STuder, Introduzione, in: Storia della teologia. I: Epoca patristica, edd. A. Di Berardino - B. Studer, Casale Monferrato (AL) 1993, 513.

${ }^{5}$ Cf. H.R. Drobner, Patrologia, Casale Monferrato (AL) 2002², 571.

${ }^{6}$ Cf. P. Parente, Teologia, in: Enciclopedia cattolica, XI, Città del Vaticano 1953, col. 1962.

${ }^{7}$ Cf. B. Altaner, Patrologia, Torino $1966^{6}, 341$.

${ }^{8}$ Cf. M. Grabmann, Storia della teologia cattolica, dalla fine dell'epoca patristica ai tempi nostri, Milano 1939², 25-26. 
subito accompagnata da quella di ferro (sec. VII) ${ }^{9}$. E del resto, a parte la rinascita carolingia del secolo IX, non venne poi rapidamente una fase di grande crescita $^{10}$.

\section{Il diritto canonico nei secoli V-VIII: la Collectio Dionysiana, Gregorio Magno, i concili}

Per il diritto canonico fu diverso, l'andamento fu differente rispetto a quello della teologia. A questo proposito, voglio mettere in risalto alcuni dati significativi che riguardano rispettivamente l'opera di Dionigi il Piccolo, Gregorio Magno e l'attività conciliare.

\subsection{L'opera di Dionigi il Piccolo}

Dionigi il Piccolo, monaco scita che dal termine del V secolo viveva a Roma, dove morì qualche decennio dopo, fece una raccolta di testi ${ }^{11}$ della quale la Chiesa Romana si servì poi costantemente ${ }^{12}$ : la Collectio Dionysiana diventò di uso universale nel medioevo ${ }^{13}$. Già prima erano state compilate raccolte di norme canoniche, ma la Collectio Dionysiana ebbe particolare valore per l'ulteriore sviluppo del diritto ${ }^{14}$. Essa inoltre assunse un'importanza non solo giuridica, ma anche politico-ecclesiale: fino ad allora il papato, godendo di una posizione di grande prestigio e giocando un ruolo primaziale in Occidente, non si era ancora dotato di un corpus di norme giuridiche tale da assumere rilievo per l'intera Chiesa; alcuni papi, come Gelasio I, avevano rivendicato una potestas suprema, pari a quella imperiale, ma Dionigi il Piccolo rese al vescovo di Roma un grande servizio, quello di dotarlo di uno strumento normativo, «che per i tempi era il massimo

\footnotetext{
${ }^{9}$ B. Mondin, Storia della teologia. I: Epoca patristica, Bologna 1996, 441.

${ }^{10}$ Cf. ibid. II: Epoca scolastica, Bologna 1996, 7.

${ }^{11}$ Cf. PL 67, coll. 27-346.

${ }^{12}$ Cf. P. De Labriolle, La vita cristiana in Occidente, in: Storia della Chiesa, dalle origini fino ai giorni nostri, edd. A. Fliche - V. Martin. IV: Dalla morte di Teodosio all'avvento di San Gregorio Magno, Torino $1961^{2}, 745$.

${ }^{13}$ Cf. A. Ferrua, L'attività letteraria della Chiesa in Italia, ibid., 774.

${ }^{14}$ Cf. K.S. Frank, Manuale di storia della Chiesa antica, Città del Vaticano 2000, 364.
} 
raggiungibile», aumentando così anche di molto il ruolo e il prestigio della Cancelleria romana, che di lì a poco, con i libri rituali ed il Liber diurnus dei pontefici costituì un obbligato punto di riferimento, sia dal punto di vista liturgico che disciplinare e giuridico ${ }^{15}$. Se prima della collezione dionisiana le raccolte di concili africane, spagnole ed italiane potevano equivalersi, ora invece le fonti normative romane si elevano nettamente per posizione e prestigio e presto anche per efficacia giuridica al di sopra delle altre ${ }^{16}$. La collezione Dionysiana costituì non solo la prima raccolta organica di canoni basata su testi autentici o generalmente ammessi e disposti secondo un certo ordine, ma anche il punto di partenza di un diritto papale che tendeva a rendersi autonomo ${ }^{17}$. In essa fu importante l'organizzazione del materiale secondo un ordine, anche con l'eliminazione di materiale regionale occidentale, come pure la distinzione tra fonti conciliari e decretali, che vennero distribuite in capitoli con propria numerazione per i diversi papi $^{18}$. Il principio nuovo della sistemazione del materiale ha influito anche sulle collezioni successive, come quella di Cresconio, la Vetus Gallica e la Hispana systematica ${ }^{19}$. La Dionysiana, per giunta, si distinse per una migliore traduzione attenta all'originale greco e una migliore redazione in latino ${ }^{20}$. È stato affermato che l'abbondanza di informazione, la buona identificazione dei testi, il desiderio di universalismo testimoniato dalla giustapposizione di testi, di origine e data molto varie, fecero per secoli della Dionysiana

${ }^{15}$ Cf. L. Musselli, Storia del diritto canonico. Introduzione alla storia del diritto e delle istituzioni ecclesiali, Torino $2007^{2}, 26-27$.

${ }^{16}$ Cf. ibid., 27.

${ }^{17}$ Cf. C. FAntappiè, Introduzione storica al diritto canonico, Bologna 1999, 67.

${ }^{18}$ Cf. A. Di Berardino, Letteratura canonistica, penitenziale e liturgica, in: Patrologia. IV: Dal Concilio di Calcedonia (451) a Beda. I Padri latini, ed. Id., Genova 1996, 504; ID., La letteratura canonico-liturgica e conciliare, in: H.R. Drobner, 617.

${ }^{19}$ Cf. A. Di Berardino, Letteratura canonistica, penitenziale e liturgica, 504; ID., La letteratura canonico-liturgica e conciliare, 617.

${ }^{20}$ Cf. ID., La letteratura canonico-liturgica e conciliare, 617. 
la più importante tra le collezioni canoniche dell'Occidente ${ }^{21}$. È stato altresì asserito che essa determinò la disciplina occidentale fino agli albori del secondo millennio ${ }^{22}$. Carlo Magno introdusse nel suo regno la raccolta di norme di Dionigi il Piccolo, a lui consegnata da papa Adriano I nel 774 e ampliata con diverse aggiunte ${ }^{23}$. Nella storiografia, circa il diritto canonico fra tardo antico e alto medioevo, si è parlato di una "svolta dionisiana" nella canonistica» ${ }^{24}$ ed è stato scritto che «Tutte le collezioni canoniche posteriori sono debitrici dell'opera di Dionigi ${ }^{25}$ e che questa ha avuto importanza decisiva per la storia delle collezioni canoniche ${ }^{26}$. Dionigi il Piccolo è stato considerato persino l'iniziatore del diritto canonico ${ }^{27}$, il padre del diritto canonico ${ }^{28}$ e almeno uno dei padri fondatori del diritto canonico ${ }^{29}$. È stato sostenuto che l'evoluzione del diritto canonico dalla sua prima origine con Dionigi, è proceduta su una linea diretta e ininterrotta fino al culmine nel Decreto di Graziano ${ }^{30}$. Tra la fine del V secolo e l'VIII secolo ci fu un nuovo, distinguibile periodo nella storia delle

${ }^{21}$ Cf. J. Gaudemet, Storia del diritto canonico. Ecclesia et civitas, Cinisello Balsamo (MI) 1998, 69 .

${ }^{22}$ Cf. G. Brugnotto, Collezioni canoniche: brani scelti. I. La collezione Dionysiana, Quaderni di diritto ecclesiale 12(1999), 404.

${ }^{23}$ Cf. K. Bihlmeyer - H. Tuechle. II: Il medioevo, ed. I. Rogger, Brescia $1987^{8}$, 124.

${ }^{24} \mathrm{Cf}$. H. Mordek, Il diritto canonico fra tardo antico e alto medioevo. La "svolta dionisiana" nella canonistica, in: La cultura in Italia fra tardo antico e alto medioevo, Roma 1981, I, 149-164.

${ }^{25}$ Cf. A. Di Berardino, Dionigi il Piccolo, in: Letteratura patristica, edd. Id. - G. Fedalto - M. Simonetti, Cinisello Balsamo (MI) 2007, 398.

${ }^{26}$ Cf. Ch. Munier, Collezioni canoniche, in: Dizionario patristico e di antichità cristiane, ed. A. Di Berardino, I, Casale Monferrato (AL) 1983, col. 734.

${ }^{27}$ Cf. V. LoI, Dionigi il Piccolo, ibid., col. 983.

${ }^{28}$ Cf. W.M. Peitz, Dionysius Exiguus - Studien. Neue Wege der philologischen und historischen Text und Quellenkritik, Arbeiten zur Kirchengeschichte, Berlin 1960, 20, cit. in: F. Duta, Des précisions sur la biographie de Denys le Petit, Revue de droit canonique 49(1999), 296.

${ }^{29}$ Cf. F. Duta, 279.

${ }^{30}$ Cf. W.M. Peitz, Gratian und Dionysius Exiguus, in: Studia gratiana, post octava Decreti sæcularia, I, edd. G. Forchielli - A.M. Stickler, Bologna 1953, 51-81. 
collezioni canoniche ${ }^{31}$, del quale la collezione di Dionigi il Piccolo costituisce un elemento di primaria importanza.

\subsection{Gregorio Magno}

Gregorio Magno ( $†$ 604), una delle figure di maggior rilievo dell'alto medioevo ${ }^{32}$, la cui personalità si eleva «imponente» alla fine dell'antichità cristiana e sulla soglia del medioevo ${ }^{33}$, «scrittore grandissimo» e papa il cui pontificato fu «uno dei più grandi della storia della Chiesa» ${ }^{34}$, ebbe sensibilità per il diritto e per il diritto canonico e fu anche importante nella storia di quest'ultimo ${ }^{35}$. Mostrò attitudine ad identificare il diritto canonico distinguendolo da quello civile ${ }^{36}$. Fece riferimento ai «canoni» e nelle fonti del diritto canonico riservò un posto particolare alle decretali dei papi suoi predecessori ${ }^{37}$. In armonia con quella che già allora poteva ritenersi la tradizione dei concili, dei Padri e, in particolare, delle decretali papali, applicò tali orientamenti ai casi concreti, tenendo presenti i principi del diritto romano e così contribuì notevolmente allo sviluppo del diritto canonico, tanto da risultare uno dei Padri della Chiesa citati con maggior frequenza nella successiva tradizione canonistica ${ }^{38}$. Nelle tavole ricomposte da Charles Munier, sulla presenza dei testi attribuiti ai Padri nelle varie collezioni canoniche e in particolare nel Decreto di Graziano, soltanto Agostino, Gerolamo e Ambrogio precedono,

${ }^{31}$ Cf. R. NAZ, Droit canonique, in: DDC, IV, coll. 1455-1458.

${ }^{32}$ Cf. V. Recchia, Gregorio Magno e la conclusione dell'età patristica in Occidente: l'antropologia soprannaturale e la contemplazione, in: Storia della teologia. I: Dalle origini a Bernardo di Chiaravalle, ed. E. dal Covolo, Roma-Bologna 1995, 427.

${ }^{33}$ Cf. K. Bihlmeyer - H. Tuechle, I, 384; K.S. Frank, 360.

${ }^{34}$ Cf. G. BARdy - G. Di NolA, Storia della letteratura cristiana antica latina. Storia letteraria, letteratura critica e approfondimenti tematici, Città del Vaticano 1999, 575.

${ }^{35}$ Cf. G. Picasso, Diritto canonico, in: Enciclopedia gregoriana. La vita, l'opera e la fortuna di Gregorio Magno, edd. G. Cremascoli - A. Degl'Innocenti, Firenze 2008, 94-95.

${ }^{36}$ Cf. ibid., 94 .

${ }^{37}$ Cf. ibid.

${ }^{38}$ Cf. ibid. 
per numero di brani citati, quelli di Gregorio Magno e, per giunta, in alcune situazioni questi acquistano anche maggior rilievo ${ }^{39}$. La collezione canonica italiana "Anselmo dedicata», compilata verso la fine del secolo IX e composta di dodici libri relativi ai vari aspetti della vita della Chiesa, in ogni suo libro, dopo una parte con testi canonistici desunti da diverse fonti, contiene sempre una sezione di brani ricavati dalle lettere di Gregorio Magno ${ }^{40}$. Il coevo Reginone di Prüm nella raccolta De synodalibus causis et disciplinis ecclesiasticis inserì brani significativi sui peccati e sulla penitenza presi dai Moralia in Job di Gregorio Magno ${ }^{41}$. Con il Decretum di Burcardo di Worms dei primi anni del secolo XI, la raccolta canonistica che ebbe la maggior diffusione prima del Decreto di Graziano, gli scritti di Gregorio Magno assunsero un particolare rilievo: specialmente i libri XIX e XX accolgono numerosi testi gregoriani, dalle lettere e dai Moralia $^{42}$. È possibile distinguere un settore del diritto canonico, allora strettamente congiunto con la teologia morale, nel quale Gregorio è autorevole maestro ${ }^{43}$. Nello sviluppo delle collezioni canoniche, da quelle dell'età di Gregorio VII fino alle raccolte di Ivo di Chartres e poi al Decreto di Graziano, i testi di Gregorio Magno sono presenti in modo significativo, desunti in massima parte dai Moralia e meno dai Dialogi e più dalle lettere ${ }^{44}$. Gli argomenti per i quali è invocata l'autorità di Gregorio Magno sono molto diversi: nelle raccolte canonistiche più antiche egli occupa un posto notevole per il suo insegnamento di carattere morale e in ordine all'escatologia; nel Decreto di Graziano egli risalta anche in relazione ai temi che la riforma della Chiesa del secolo XI aveva reso di particolare attualità, come la libertà dei monaci, la figura del vescovo, la simonia ${ }^{45}$.

\footnotetext{
${ }^{39}$ Cf. ibid.

${ }^{40}$ Cf. ibid., 95.

${ }^{41}$ Cf. ibid.

${ }^{42}$ Cf. ibid.

${ }^{43}$ Cf. ibid.

${ }^{44}$ Cf. ibid.

${ }^{45}$ Cf. ibid.
} 


\subsection{I concili}

Dal V al X secolo l'Occidente conobbe un'intensa attività conciliare, dapprima in Gallia e nella Spagna visigota, poi nell'impero carolingio $^{46}$. Nelle Chiese nazionali dei Merovingi e dei Visigoti furono, nella maggior parte dei casi, i concili a stabilire la disciplina, a giudicare le deviazioni e in alcuni casi a ricordare la vera dottrina ${ }^{47}$. I concili tra i loro compiti avevano quello legislativo e in tale periodo ebbero un ruolo importante nel «dire il diritto» ${ }^{48}$. I concili hanno organizzato e retto le Chiese di Gallia nel VI e VII secolo, hanno stabilito il diritto della Chiesa visigota dall'inizio del VI secolo fino alla conquista araba del 711, a metà dell'VIII secolo hanno gettato le basi della riforma della Chiesa carolingia ${ }^{49}$. In Gallia nel VI e VII secolo la Chiesa mostrò vitalità con la celebrazione di concili e la promulgazione di canoni ${ }^{50}$. Nella Spagna visigotica tra la fine del secolo VI e l'inizio del secolo VIII si verificò una notevole fioritura dell'attività conciliare e risulta che in 114 anni siano stati celebrati 28 concili, dei quali 18 a Toledo e i rimanenti in altre città ${ }^{51}$. Tali concili rappresentano l'istituzione più caratteristica della Chiesa visigota, essendo l'asse attorno al quale si articolò tutta la sua vita religiosa ${ }^{52}$. In essi veniva definita la dottrina, si regolavano le relazioni con lo Stato e si stabilivano e modificavano le norme che regolavano la vita ecclesiale interna, la disciplina dei religiosi, la morale dei fedeli e le norme liturgiche ${ }^{53}$. I concili esercitarono anche un influsso sulla monarchia e sul governo visigotici ${ }^{54}$. È stato osservato che la letteratura

\footnotetext{
${ }^{46}$ Cf. J. Gaudemet, 258.

${ }^{47}$ Cf. ibid.

${ }^{48}$ Cf. ibid., 207.

${ }^{49}$ Cf. ibid.

${ }^{50}$ Cf. Concilia Galliae, A. 511 - A. 695, ed. C. de Clercq, Turnholti 1963 (CCSL $148 \mathrm{~A})$.

${ }^{51}$ Cf. J.M. LaboA, Atlante dei concili e dei sinodi nella storia della Chiesa, Milano-Roma 2008, 76.

${ }^{52}$ Cf. ibid., 77.

${ }^{53}$ Cf. ibid.

${ }^{54}$ Cf. ibid., 79.
} 
ecclesiastica di tarda età visigotica pare caratterizzata soprattutto da scarsa originalità e superficialità di indagine e che a tale decadenza fanno eccezione l'interesse per l'efficacia regolativa del diritto canonico e alcuni sviluppi della produzione letteraria legata all'ambito monastico, nella quale, inoltre, è compreso il genere normativo ${ }^{55}$. In Gallia nei secoli VI-VII si tennero almeno 55 concili $^{56}$. I canoni di essi stabilirono i doveri e formularono i divieti, riguardarono aspetti della vita quotidiana delle comunità, dei chierici e dei laici, dei vescovi e dei monaci, aspetti della vita liturgica e sacramentale e delle relazioni con il potere; le norme conciliari venivano accompagnate dalla disposizione delle pene nel caso non fossero rispettate; gli ecclesiastici costituivano l'oggetto preponderante ${ }^{57}$. Canoni di concili merovingi sono stati recepiti nelle collezioni canoniche posteriori, incluso il Decreto di Graziano ${ }^{58}$. Importanti furono anche i concili di S. Bonifacio nell'VIII secolo ${ }^{59}$. E bisogna ancora aggiungere che, a quanto pare, il secolo VI fu fondamentale nella storia dei sinodi diocesani ${ }^{60}$.

${ }^{55}$ Cf. G. D'Onofrio, Il rinascere della Christianitas (secoli VI-VIII), in: Storia della teologia nel medioevo, ed. Id. I: I princìpi, Casale Monferrato (AL) 1996, 84-85.

${ }^{56}$ Cf. J.M. LABOA, 80.

${ }^{57}$ Cf. ibid., 80-81.

${ }^{58} \mathrm{Cf}$. Les canons des conciles mérovingiens (VI $\mathrm{e}^{\mathrm{e}}-\mathrm{VII} \mathrm{e}^{\mathrm{e}}$ siècles), texte latin de l'édition C. de Clercq, introduction, traduction et notes par J. Gaudemet et B. Basdevant, Paris 1989, 2 voll. (SCh 353-354), I: 13-32 e 67-345 passim nelle note; II: 346-591 passim nelle note e 625-629.

${ }^{59}$ Cf., p. es., K. Bihlmeyer - H. Tuechle, II, 32-33; J.M. Laboa, 102-105.

${ }^{60} \mathrm{Cf}$. G. Corbellini, Il sinodo diocesano nel nuovo Codex iuris canonici, Roma 1986, 18-21; O. Pontal, Évolution historique du synode diocésain, in: La synodalité. La participation au gouvernement dans l'Église. Actes du VII e congrès international de droit canonique, Paris, Unesco, 21-28 septembre 1990, L’année canonique, hors série, 1992, II, 524-525. 
In Oriente fu importante l'opera canonica del Concilio Trullano del $692^{61}$, le norme emanate dal quale sarebbero divenute «la principale fonte del diritto canonico della Chiesa bizantina» ${ }^{62}$.

\section{Presenza del diritto e tendenze al giuridismo nella Chiesa dei secoli V-VIII: le regole monastiche, i penitenziali}

Nella comunità ecclesiale dei secoli V-VIII si trovano, poi, presenze varie del diritto. Interessante è anche il caso del diritto interno del monachesimo ${ }^{63}$. Nel VI secolo si colloca la nascita della regola di S. Benedetto, dopo che dal V secolo aveva avuto inizio «una ricca produzione di regole latine», che intendevano consolidare la tradizione monastica e la sua pratica per il singolo monastero ${ }^{64}$. Venne pure la regola di S. Colombano ${ }^{65}$. Tra il V e il VII secolo furono composte «tante» regole ${ }^{66}$. La regola benedettina, come è noto, ha avuto un ruolo importantissimo nella storia del monachesimo in Occidente e nella storia della Chiesa ${ }^{67}$. È stato sostenuto che Benedetto, con la sua regola, ripropose in un momento di crisi e di travaglio profondi di tutto il mondo occidentale un codice spirituale che presentava un orizzonte di universalità e che riuscì a comporre la grande tradizione anche giuridica romana con l'esperienza cristiana aperta agli apporti dei popoli dell'Est e del Nord $^{68}$. Ed è stato scritto che la regola di $\mathrm{S}$. Benedetto fu frutto dello spirito giuridico romano, che con senso pratico e talento organizzativo seppe conferire alla pianta orientale

${ }^{61}$ Cf. L. BréHIER, La vita della Chiesa bizantina da Maurizio a Costantino V, in: Storia della Chiesa, dalle origini ai nostri giorni, edd. A. Fliche - V. Martin. V:

L. Bréhier - R. Aigrain, San Gregorio Magno, gli Stati barbarici e la conquista araba (590-757), Torino 1971², 627-628; L. MusselLI, 27.

${ }^{62}$ Cf. L. BRÉHIER, 627.

${ }^{63} \mathrm{Cf}$. analogamente J. Gaudemet, Storia del diritto canonico, 208.

${ }^{64}$ Cf. K.S. Frank, 415.

${ }^{65}$ Cf. M. Dell'Omo, Storia del monachesimo occidentale dal medioevo all'età contemporanea. Il carisma di San Benedetto tra VI e XX secolo, Milano 2011, 6-7.

${ }^{66}$ Cf. ibid., 4. Cf. anche S. Pricoco, Introduzione, in: La regola di San Benedetto e le regole dei Padri, ed. Id., s.1. 2007 ${ }^{5}$, IX-XII.

${ }^{67}$ Cf., p. es., K. Bihlmeyer - H. Tuechle, I, 443.

${ }^{68}$ Cf. P. Siniscalco, S. Benedetto, in: Patrologia, IV, 234. 
una forma e una struttura corrispondenti alle diverse condizioni dell'Occidente ${ }^{69}$.

Nella Chiesa dei secoli V-VIII emersero inoltre tendenze al giuridismo. È sintomatico il caso dei penitenziali, che si diffusero dal VI secolo in Occidente. Come noto, essi erano cataloghi più o meno lunghi, destinati ai confessori, di atti considerati peccati con le rispettive penitenze espiatorie: per ogni peccato veniva precisata la penitenza ${ }^{70}$. Contenevano elenchi di peccati e, per ciascun peccato, l'indicazione di una penitenza o di un'ammenda che doveva essere inflitta al peccatore ${ }^{71}$. Il metodo era casistico ${ }^{72}$. Non avevano carattere descrittivo ma normativo, talvolta persino quasi meccanico ${ }^{73}$. È stato affermato che l'amministrazione della penitenza risultò organizzata «in uno stile giuridico, come un processo in cui il confessore è il giudice che applica non leggi generali ma specifiche e precise norme per i singoli casi analogamente a quanto avviene nei tribunali secolari» ${ }^{74}$. I penitenziali inaugurarono un genere letterario che, pur con differenze, sarebbe stato continuato con le somme medievali e con i casi di coscienza in età moderna ${ }^{75}$. Ebbero «una grande importanza» per l'evoluzione del diritto penale canonico ${ }^{76}$ e svolsero un ruolo, p. es., per la disciplina canonica del matrimonio ${ }^{77}$.

\footnotetext{
${ }^{69}$ Cf. B. Altaner, 359.

${ }^{70}$ Cf. A. Di Berardino, Letteratura canonistica, penitenziale e liturgica, 515.

${ }^{71}$ Cf. P.J. Geary, Penitenziali. Occidente, in: Dizionario enciclopedico del medioevo, ed. A. Vauchez, III, Roma 1999, 1441.

${ }^{72}$ Cf. L. Musselli, 31.

${ }^{73}$ Cf. A. Di Berardino, Letteratura canonistica, penitenziale e liturgica, 515.

${ }^{74}$ Cf. P. Prodi, L'istituto della penitenza: nodi storici, Chiesa e storia 1(2011), 20.

${ }^{75}$ Cf. C. FAntappiè, 73.

${ }^{76}$ Cf. L. Musselli, 31.

${ }^{77}$ Cf. E. GRILlo, Il matrimonio nei penitenziali altomedievali: alle origini della disciplina canonica, Il diritto ecclesiastico 115(2004), I, 950-1007.
} 


\section{Osservazioni conclusive}

Il periodo compreso tra i secoli V e VIII è stato importante nella storia del diritto canonico ${ }^{78}$. Per giunta, è stato importante nella storia del diritto canonico sotto vari profili: quello delle raccolte di testi, quello delle istituzioni, quello del progresso scientifico, quello dell'incremento delle norme. Fu allora che il diritto canonico ebbe quegli sviluppi che furono importanti per la funzione che - secondo quanto è stato sostenuto nella storiografia - svolse nella formazione dell'Europa $^{79}$. In tale periodo, del resto, si collocano fatti notevolmente rilevanti pure per la storia del diritto in generale, come l'opera legislativa dell'imperatore Giustiniano $\mathrm{I}^{80} \mathrm{e}$ la penetrazione del germanesimo verso Occidente ${ }^{81}$, le quali, tra l'altro, hanno avuto grande importanza anche per il diritto canonico: quest'ultimo nel VI secolo era ancora poco costituito in Oriente e la legislazione di Giustiniano andò a colmare lacune ${ }^{82}$; nel campo della costituzione ecclesiastica e del diritto canonico l'influsso delle concezioni e delle istituzioni germaniche fu forte ${ }^{83}$. Quello racchiuso tra i secoli V e VIII fu un periodo complesso pure per il diritto canonico: se le collezioni di Dionigi il Piccolo sembrano dover molto alla tecnica del Codice teodosiano, a quanto pare la conoscenza del diritto romano e la formazione giuridica pressoché sparirono nei regni barbarici succeduti all'impero romano, con l'eccezione costituita dalla Spagna visigotica, dove la cultura giuridica ha dato un valido contributo

\footnotetext{
${ }^{78}$ Cf. anche P. Riché, Da Gregorio Magno a Pipino il Breve (dal secolo VII alla metà del secolo VIII), in: Storia del cristianesimo. Religione-politica-cultura. IV: Vescovi, monaci e imperatori (610-1054), edd. G. Dagron - P. Riché - A. Vauchez, Roma 1999, 688-689.

${ }^{79}$ Cf. D. Composta, Il diritto canonico nella formazione dell'Europa (325-800 d. Cr.), Apollinaris 62(1989), 597-621.

${ }^{80}$ Cf., p. es., E. Albertario, Corpus iuris civilis, in: Enciclopedia cattolica, IV, Città del Vaticano 1950, coll. 622-629.

${ }^{81}$ Cf. F. Calasso, Medio evo del diritto. I: Le fonti, Milano 1954.

${ }^{82}$ Cf. L. BréHIER, La vita cristiana in Oriente, in: Storia della Chiesa, dalle origini fino ai giorni nostri, edd. A. Fliche - V. Martin, IV, 673.

${ }^{83}$ Cf. K. Bihlmeyer - H. Tuechle, II, 123-125; C. Fantappiè, 74-79.
} 
anche alla vita della Chiesa e - altro dato interessante riguardante il periodo in questione - S. Isidoro di Siviglia, nelle sue Etimologie, ha gettato le basi del pensiero giuridico ecclesiale medievale ${ }^{84}$. Ė stato scritto che, da una parte la traduzione in latino delle collezioni dei canoni conciliari orientali e la raccolta delle decretali papali del secolo $\mathrm{V}$, che hanno meritato a Dionigi il Piccolo il titolo di «padre della canonistica», dall'altra il grande compendio eclettico della dottrina giuridica cristiana precedente fatto da Isidoro di Siviglia ( $†$ 636) nelle Etymologiae, in cui, oltre alle sentenze dei Padri della Chiesa, vengono per la prima volta ampiamente elaborate quelle dei giureconsulti romani, e, da ultimo, l'impatto del pensiero cristiano con lo spirito del diritto germanico, hanno segnato profondamente la fine dell'antichità classica e l'inizio del primo medioevo ${ }^{85}$. Comunque, per il diritto canonico non si è trattato di un tempo di transizione, ma di una fase ampiamente fondante.

Ad ogni modo, nel periodo compreso tra i secoli V e VIII, diritto canonico e teologia ebbero un diverso andamento. Complessivamente, se nella teologia si verificò un certo ripiegamento, nel diritto canonico sussistettero elementi di notevole crescita e di espansione. Di fronte a ciò, è necessario tenere conto delle peculiarità delle diverse discipline. La differenza inoltre potrebbe non apparire così strana considerandola in una chiave interpretativa storico-psicologica. La medesima tendenza conservativa che frenava la teologia e la distoglieva dagli slanci poteva forse dare impulso allo sviluppo del diritto canonico. Il diritto canonico, in tal senso, poteva essere la risposta strumentale ad una ricerca di stabilizzazione. Un'insicurezza e un'aspirazione al consolidamento potrebbero, da una parte, aver frenato la creatività della teologia e, dall'altra, aver favorito una crescita del diritto canonico.

\footnotetext{
${ }^{84}$ Cf. P. Erdö, Storia del diritto canonico. Disciplina, in: Nuovo dizionario di diritto canonico, edd. C. Corral Salvador - V. De Paolis - G. Ghirlanda, Cinisello Balsamo (MI) $1996^{2}, 1041$.

${ }^{85}$ Cf. E. Corecco, Diritto, in: Dizionario teologico interdisciplinare, I, Torino 1977,123 , ora anche in ID., Ius et communio. Scritti di diritto canonico, edd. G. Borgonovo - A. Cattaneo, I, Casale Monferrato (AL) - Lugano 1997, 85.
} 
Nella Chiesa del tempo apparirebbero inclinazioni alla meticolosità, che avrebbero favorito la tendenza alla conservazione, all'erudizione, al diritto e al giuridismo. La tendenza a raccogliere, custodire e trasmettere poté forse produrre esiti diversi rispettivamente nel campo della teologia e in quello del diritto canonico. Sono tuttavia solo ipotesi, per di più con i limiti propri di congetture fondate su di un semplice sguardo generale. Ė certamente necessaria una disamina più concreta e puntuale, prospettica e insieme calata nella storia $\mathrm{e}$ nel contesto. Particolarmente in Occidente, forse poterono giocare a favore del diritto canonico pure i resti del pragmatismo del mondo romano e l'attitudine pratica dei popoli germanici. Per non dire che, ovviamente, tutto era, in fin dei conti, legato anche alle capacità, alle qualità e alle caratteristiche degli uomini che vi erano in quella fase storica.

Ma bisogna tenere presente un'altra cosa. Ho parlato distintamente di teologia e di diritto canonico, però il diritto canonico non è estraneo alla teologia e anzi, almeno da un certo punto di vista, vi è compreso ${ }^{86}$. Mi chiedo allora se, tenendo conto del diritto canonico, si debba rettificare in qualche misura il bilancio generale della produzione teologica dei secoli V-VIII e, quanto meno, se non sia opportuna una visione contemporaneamente più panoramica ed articolata.

Queste sono le suggestioni di fondo che emergono dalla mia riflessione. Il punto di arrivo di essa è solo un eventuale punto di partenza per verifiche e approfondimenti.

\section{Canon law in the context of the theology and Church life from V to VIII century}

In the period between the 5th and 8th centuries, Canon Law and Theology followed a different course. While Theology was experiencing a sort of regression, Canon Law showed elements of remarkable growth and expansion. The article highlights the work of Dionysius Exiguus, the figure of

\footnotetext{
${ }^{86}$ Cf., p. es., A.M. Stickler, Teologia e diritto canonico nella storia, in: Teologia e diritto canonico, Città del Vaticano 1987, 20.
} 
Gregory the Great, as well as councils, jurisprudence and tendencies to legalism in the Church from the 5th to the 8th century. In the end, it is suggested that, taking account of Canon Law, it might be correct to rethink the overall evaluation of the theological production of that time.

Parole chiave: diritto canonico secoli V-VIII, teologia secoli V-VIII, Chiesa secoli V-VIII

KeYwords: Canon law century V-VIII, Theology century V-VIII, Church century V-VIII

\section{BREVE NOTA SULL'AUTORE:}

Aldo Gorini - Docente di Storia della Chiesa e di Metodologia teologica e bibliotecario dell' Istituto Superiore di Scienze Religiose di Genova; professore a contratto del Dipartimento di Scienze Politiche dell'Università degli Studi di Genova; responsabile della Biblioteca Diocesana «Mons. Luigi Roba» e bibliotecario della Sezione della Facoltà Teologica dell'Italia Settentrionale, presso il Seminario Arcivescovile «Benedetto XV» di Genova. 